\title{
De la performativité de l'image dans une phénoménologie non intentionnelle
}

\section{Sylvaine Gourdain}

\section{(2) OpenEdition}

1 Journals

Édition électronique

URL : https://journals.openedition.org/alter/1912

DOI : 10.4000/alter.1912

ISSN : 2558-7927

Éditeur :

Association ALTER, Archives Husserl (CNRS-UMR 8547)

\section{Édition imprimée}

Date de publication : 1 novembre 2019

Pagination : 173-188

ISBN : 978-2-9550449-5-7

ISSN : $1249-8947$

\section{Référence électronique}

Sylvaine Gourdain, "De la performativité de l'image dans une phénoménologie non intentionnelle », Alter [En ligne], 27 | 2019, mis en ligne le 22 décembre 2020, consulté le 13 juin 2021. URL : http:// journals.openedition.org/alter/1912; DOI : https://doi.org/10.4000/alter.1912

Ce document a été généré automatiquement le 13 juin 2021.

Revue Alter 


\title{
De la performativité de l'image dans une phénoménologie non intentionnelle
}

\author{
Sylvaine Gourdain
}

\section{Introduction : De la performativité des actes de parole selon Austin à la performativité de l'image}

1 On observe aujourd'hui dans la recherche en sciences humaines une utilisation de plus en plus fréquente du lexique de la «performativité » et ce dans des sens très divers, depuis l'usage hérité de John L. Austin, qui a inventé le terme dans son livre Quand dire c'est faire ${ }^{1}$, jusqu'aux emplois en sociologie ${ }^{2}$, en théorie du genre ${ }^{3}$, dans les études théâtrales ${ }^{4}$ ou en esthétique ${ }^{5}$. Rappelons d'abord que pour Austin, un énoncé performatif est un énoncé qui réalise l'action qu'il exprime, comme dans le cas : «Je baptise ce bateau le Queen Elizabeth ${ }^{6}$. Ce type d'énoncé - comme l'acte de baptiser, une promesse, ou encore un pari - ne se contente pas de décrire ou de constater, mais il exécute une action par le fait même de la prononcer : il fait en disant. Austin détermine un certain nombre de critères nécessaires à la réussite d'un énoncé performatif comme l'existence d'une procédure conventionnelle ${ }^{7}$. De plus, selon lui, ces énoncés ne sont ni vrais ni faux, mais ils réussissent ou ils échouent. Il semble ainsi que l'on oscille entre une utilisation du terme de "performatif» en philosophie du langage et en linguistique, qui reprend à la lettre la définition austinienne, et un usage tout à fait différent où les thèses du philosophe anglais n'interviennent pas toujours et où le mot de "performatif» est employé comme synonyme de "faire advenir une réalité", « avoir des effets », «transformer le réel », ou bien par assimilation avec le terme de "performance ». Autrement dit, d'un côté semble prédominer une compréhension rigide du concept, qui, à force de coller à une définition déjà prête, risque de ne faire que l'appauvrir, alors que de l'autre, on en élargit le sens au point de tomber parfois dans des utilisations très floues et vagues. 
2 Nous voudrions pour notre part développer une voie intermédiaire, en proposant ici une conception de la performativité de l'image dans le cadre d'une phénoménologie non intentionnelle. Cette conception entend éviter les dangers inhérents aux deux usages les plus extrêmes qui sont faits du lexique de la performativité, à savoir un usage trop étroit et trop figé ou un usage trop imprécis et trop lâche. Nous verrons que le terme de " performatif » est indispensable pour définir le type d'image dont il s'agit ici, même si l'utilisation qui en est faite n'est pas austinienne. De plus, soutenir une performativité de l'image n'est pas soumettre l'image aux règles du langage et retomber ainsi dans une compréhension purement sémiologique de l'image. Toutefois, nous ne nous interdirons pas, bien au contraire, de reprendre certains critères de la performativité des actes de parole déterminés par Austin. Telle est selon nous la tâche de la philosophie: déployer les concepts d'une nouvelle manière et leur offrir un héritage fructueux en prolongeant leur pertinence sans les priver de leur sens. C'est pourquoi il nous semble que, même si le performatif austinien est un concept seulement provisoire, qu'Austin préfère finalement abandonner - puisqu'il reconnaît en définitive que toute énonciation est plus qu'une simple description et a des effets sur le réel - il est tout à fait légitime d'en faire usage à nouveaux frais, en dépit ou au-delà d'Austin. En ce sens, l'image peut être une manière de redonner un sens spécifique et singulier au terme de "performatif ", qu'aucun autre concept ne permet d'approcher, autrement dit de rendre au performatif sa pertinence propre, et par là même, son caractère incontournable. Nous montrerons ainsi qu'une telle conception de l'image performative permet de repenser l'apparaître hors de la polarisation sujet-objet et hors toute intentionnalité comprise comme rapport au monde d'emblée orienté vers quelque chose.

\section{Le faire-image, une nouvelle forme de parole}

3 Il est nécessaire d'abord de revenir sur le type d'image auquel nous faisons référence ici. Qu'il s'agisse en effet de l'image "dans une phénoménologie non intentionnelle » n'est ni contingent, ni accidentel. Au contraire, l'image telle que nous la concevons ne peut se déployer que dans une phénoménologie non intentionnelle, précisément parce qu'elle est en elle-même ce par quoi l'intentionnalité est ébranlée : avant de s'incarner en un objet physique comme un tableau ou une image cinématographique, l'image désigne la modalité la plus originaire de notre rapport au monde, une modalité qui est, par définition - nous allons nous en expliquer -, non intentionnelle. L'image qui est ici en question n'est donc compatible ni avec l'idée husserlienne de " conscience d'image » (Bildbewusstsein), ni avec celle d'«imagination» (Phantasie), qui toutes deux sont des actes intentionnels. Dans le cas de la " conscience d'image », qui s'appuie sur la triade « chose-image » (Bildding) - par exemple un tableau comme chose physique -, « objetimage » (Bildobjekt) - l'image dans son apparition spécifique d'image -, « sujet-image » (Bildsubjekt) - ce qui est visé à travers l'« objet-image »-, je vois certes dans un objet perceptif (le tableau), mais l'image qui est en jeu dans cet apparaitre perceptif est bien un apparaitre non perceptif. L' « imagination " comme " phantasia " (Phantasie) exclut quant à elle d'emblée toute donnée perceptive: selon Husserl, quand j'imagine la licorne, je la vois certes comme si je la voyais dans le monde perceptif, mais seulement parce qu'elle est entièrement absente de ce monde perceptif, toute entière nonprésente. 
4 L'image telle qu'elle est entendue ici ne peut pas être l'objet d'une visée, et elle n'est pas fictive ou imaginaire à la différence de toute présence perçue « en chair et en os ». Au contraire, l'image est pour nous une modalité originaire de notre rapport au monde en tant qu'elle est le lieu (et non la visée ou l'objet de cette visée) d'un être-au-monde sensible et en même temps herméneutique, un lieu de part en part réel. Sans être nécessairement un objet physique, l'image est réelle. Si l'image n'est pas prioritairement ou exclusivement l'objet physique «image » en tant que cet objet physique, il n'en demeure pas moins que certaines images artistiques mettent en scène de manière particulièrement appropriée l'être-au-monde qu'incarne l'image au sens qui est privilégié ici. Elles sont en quelque sorte des images au carré. Certains phénoménologues, comme Merleau-Ponty ou Maldiney, peuvent nous aider à le montrer, c'est pourquoi nous aurons aussi recours à leurs descriptions et à leurs interprétations de certaines images concrètes et notamment picturales.

Or, l'une des caractérisations principales de l'image ainsi conçue est sa performativité. Afin d'élucider en quoi consiste la performativité de l'image, ce que l'image fait, ce qu'elle nous fait, mais aussi ce qu'elle dit - puisque la performativité s'appuie sur l'unité ou le recouvrement d'un dire et d'un faire -, nous proposons pour commencer de revenir sur une citation de Derrida dans La vérité en peinture. Plus exactement, il s'agit d'une célèbre citation du peintre Cézanne, qui écrivait à Émile Bernard le 23 octobre 1905, peu de temps avant sa mort: "Je vous dois la vérité en peinture et je vous la dirai. $»^{8}$. Derrida voit dans cette phrase une double performativité : celle de l'acte de parole d'une promesse - je vous promets que je vous dirai la vérité en peinture -, qui répond à un devoir, celui de rendre un dû, de s'acquitter d'une dette - « je vous dois... » -, mais aussi celle d'un dire non constatif, « dire la vérité en peinture ». Le " promis de la promesse ", explique Derrida, "c'est un autre performatif, un "dire" qui pourrait bien, nous ne le savons pas encore, être un "peindre" qui ne dise ni ne décrive etc. ${ }^{9}$. «Dire la vérité en peinture » ne signifie pas « décrire la vérité de la peinture », comme si cette vérité était donnée et qu'il ne suffisait plus qu’à la dépeindre. «Dire la vérité en peinture " serait plutôt "dire en peinture la vérité ", et le deuxième performatif résiderait alors dans ce " dire en peinture».

On constate ici un certain déplacement du performatif, puisque l'enjeu ne repose plus tant sur le faire que sur le dire : selon toute attente et sans surprise, le peintre Cézanne s'apprête à faire de la peinture, mais il s'engage aussi - et c'est plus étonnant - à dire, et à dire la vérité. Et pourtant, par là même, il entend renoncer à toute peinture discursive, à toute image soumise à la sémiologie du langage. Ce dire doit donc être un dire proprement pictural, un dire qui émerge de la peinture, qui n'existe pas avant elle, mais que la peinture doit faire advenir, une nouvelle forme de dire que la peinture doit créer. Ainsi, pour qu'il y ait performatif, pour que la performativité de l'image picturale fonctionne, il faut non pas que le dire soit un faire, mais que le faire soit un dire et que ce dire soit engendré du faire lui-même, qu'il en prenne la forme singulière et les traits particuliers. Toutefois, l'évidence apparente du faire doit elle aussi être remise en question, car il n'est pas du tout certain que faire de la peinture s'identifie purement et simplement à faire en peinture, et c'est bien ce faire en peinture qui nous importe. Le performatif de l'image picturale selon Cézanne-Derrida pourrait donc s'énoncer ainsi : quand faire en peinture, c'est dire la vérité. D'où l'on peut extraire la formulation du performatif de l'image selon notre définition de l'image comme modalité d'être-aumonde : quand faire en image, c'est dire la vérité. 
7 Si Austin reconnaissait finalement que tout dire est un faire, ce qui rendait problématique la distinction entre énoncés performatifs et énoncés constatifs, on ne peut certes pas inverser la proposition et affirmer que tout faire est un dire. Mais on peut montrer, ce qui est notre but dans cette contribution, que le faire-image doit advenir comme un dire la vérité. On constate d'emblée ici une différence majeure avec la conception austinienne, puisque, selon Austin, un énoncé performatif n'est ni vrai ni faux. Or, il s'agit bien ici de vérité, en un sens qui va peu à peu se clarifier. Plus exactement, on peut dire que la performativité de l'image comme modalité de rapport au monde est la promesse d'un faire-image qui, en faisant, dit la vérité : l'image fait et se fait image en disant la vérité du rapport au monde qu'elle est, voilà ce que promet l'image. Mais comme promesse, elle peut échouer, et un certain échec est même, on le verra, intrinsèque à sa performativité. Nous allons donc, pas à pas, tenter de montrer en quoi consiste le « faire en peinture » et ce que signifie ce « dire la vérité ».

Tout l'enjeu du performatif de l'image, selon nous, est essentiellement lié à cette nouvelle forme de parole, à cette autre manière de parler, de dire, et donc, de faire de la philosophie. Dire par l'image ne peut pas consister à analyser quelque chose qui serait déjà achevé et que nous n'aurions plus qu'à expliciter, mais c'est faire émerger du sens, faire naître un regard, faire advenir une pensée, qui se réalise dans le dit luimême, mais un dit non clos et non définitif, qui est appelé à être prolongé dans d'autres dires, d'autres temporalités et d'autres espaces. Dire par l'image ne consiste pas à décomposer un sens déjà fixé, qui viendrait comme s'échouer sur les rives de son élucidation, mais à composer un sens dont la fécondité est précisément son mouvement permanent, sa fluidité, son ouverture. Tel est pour nous la richesse, la fécondité et le sens même de la philosophie : elle ne s'épuise pas dans le dire qui la formule, mais au contraire, elle s'ouvre en lui, elle y prend son élan. La forme d'un tel dire ne se sépare pas de son fond : dire par l'image, c'est aussi donner la parole à l'image.

\section{Recréer la vérité de notre image originaire}

Notons d'abord que la performativité délivre l'image d'une fonction de référentialité à laquelle on a longtemps, historiquement, cherché à la réduire. L'image ne re-présente pas la réalité, et elle ne décrit pas le réel pas plus qu'elle ne l'illustre, parce que, tout simplement elle ne s'y réfère pas. De même que l'image ne doit pas être subordonnée au discours, elle n'est pas un signe renvoyant à un réel qui existerait hors d'elle et qui, en elle, n'apparaîtrait que sous une forme voilée et toujours faussée, parce que médiatisée en un tiers. L'image performative est autonome et l'image est le réel, elle est le réel du rapport au monde qu'elle exprime et incarne. Nous nous appuyons ici sur une certaine conception de l'image que l'on trouve notamment chez le philosophe espagnol José Ortega y Gasset. Dans un texte datant de $1925^{10}$, Ortega évoque ce qu'il nomme "l'intracorps ", qui n'est plus le prolongement d'une conscience constituante, mais qui se rapproche plutôt du corps à la fois voyant et visible que décrit Merleau-Ponty dans ses textes tardifs. Ortega dit de l'intracorps qu'il est image, non cependant une image visible de l'extérieur, "un objet visuel», mais une image du dedans, une "image interne » " que nous charrions toujours avec nous et qui est comme le cadre au-dedans duquel tout nous apparaît $»^{11}$. Cette image n'est pourtant pas la représentation que l'on aurait, à l'intérieur, de ce qui se passe à l'extérieur, et elle n'est pas une image mentale ou d'ordre psychologique, mais elle incarne, au sens propre, elle incarne «les actions 
corporelles $»^{12}$ que je fais et que je suis tout à la fois. Cette vue du dedans est précisément l'image agissante que je suis à moi-même et au monde, et que l'on ne peut pas séparer de ce qui se passe, de ce qui se fait, dans le monde, dans le réel, puisqu'elle est aussi « le cadre au-dedans duquel tout nous apparait ».

C'est cette "image interne » - que nous nommerons désormais «image originaire ", puisqu'elle est la manière la plus fondamentale dont nous sommes au monde - que certains tableaux contribuent à réveiller, à rendre vivante, si l'on en croit les descriptions de Merleau-Ponty, mais aussi de Maldiney. Et c'est en cela que ces images picturales exhibent la vérité de notre être-au-monde, parce que, relevant de «notre insertion première dans le monde et dans le vrai ${ }^{13}$, elles l'expriment sur la toile. Or, cette «insertion première [...] dans le vrai » est « un rapport charnel au monde et à autrui $»^{14}$ : ainsi, la vérité qu'énonce la peinture, à sa manière, est une vérité qui dit l'être du sensible et son mode de fonctionnement. Mais, disant cette vérité, l'image ne produit pas un discours conceptuel sur cette vérité, elle la met en scène, elle la met en image dans sa dynamique interne, autrement dit, dans l'image, cette vérité se fait, performativement. La vérité originaire, pour Merleau-Ponty, se joue sur le plan de la « chair » du monde, c'est-à-dire de l'étoffe qui constitue le monde et autrui tout autant que notre propre corps. Car nous ne sommes voyants que parce que nous sommes visibles, nous ne pouvons être un foyer de la vision que parce que nous faisons partie du visible au même titre que les choses. Et c'est bien notre participation primordiale au monde que la peinture a la charge de mettre en œuvre et non de théoriser.

11 Le "faire en peinture", qui est mise-en-image de la vérité, se distingue donc fondamentalement d'un " faire de la peinture ", entendu comme un acte subjectif initié par l'artiste, si bien que le performatif de l'image n'est pas le performatif d'un acte ${ }^{15}$. Si le faire-image suppose l'agir de l'artiste, cet agir doit se comprendre plutôt comme une implication dans l'image et non comme la production subjective d'une image. La création dans l'image ne s'identifie pas à l'acte d'un sujet qui serait capable de fabriquer, tel un magicien, les éléments du réel, de les faire advenir ex nihilo. Pour Maldiney, un tel acte n'aboutirait qu'à un tableau raté, ce qui signerait non seulement l'échec, mais la faute de l'artiste, car la création artistique a pour lui des implications éthiques essentielles. Il cite ainsi Bonnard qui regrette de s'être « laissé aller à peindre des roses ${ }^{16}$ en voulant restituer scrupuleusement le bouquet qu'il voyait devant lui, sur la table : «je me suis laissé absorber par les détails $»^{17}$, avoue Bonnard alors qu'il lui aurait fallu laisser les détails, résister au spectacle de la vue, et garder sa vision initiale, c'est-à-dire - nous l'ajoutons - demeurer dans l'image depuis laquelle il habitait initialement le monde, demeurer dans cette « image interne » qu'évo-

quait Ortega. Le peintre doit donc d'abord oublier ce qu'il a vu face à lui, ce que sa perception a constitué, pour rendre visible ce qui se voit en lui, c'est-à-dire aussi ce que le monde dit de soi en s'infiltrant dans le peintre. C'est en cela que " [1]'art est la vérité du sensible $»^{18}$, parce qu'il recrée la «vision interne» originaire, il recrée l'image originaire que nous sommes en étant au monde et à autrui.

12 Ainsi, au lieu de viser à dessiner l'objet de son acte perceptif, l'artiste doit chercher à exprimer « ces sensations confuses que nous apportons en naissant $»^{19}$, ce que Bonnard nomme aussi la "première séduction", c'est-à-dire ce qui se forme avant toute objectivation dans notre contact avec le monde, « comme lorsqu'au détour d'une rue, un visage, une voix, une flaque de soleil sur un mur ou le courant du fleuve, déchirant tout d'un coup la pellicule de notre film quotidien, nous font la surprise d'être et d'être 
là $»^{20}$. Si nous sommes séduits, c'est bien que notre contact premier avec le monde est traversé d'une dimension pathique essentielle, et c'est cette dimension pathique, entre autres, qui empêche que le performatif de l'image soit celui d'un acte subjectif. Ces sensations, explique encore Maldiney, sont " une manière de vivre avec le monde ${ }^{21}$ et si elles sont confuses, c'est parce qu'elles n'ont pas encore été objectivées par la vie pratique. Elles expriment la vérité de notre être-au-monde le plus brut ainsi que la "virginité du monde "22 $^{22}$ selon l'expression de Cézanne, virginité qui ne désigne pas un monde sans être humain, mais un monde avec l'être humain, et non pour lui ou devant lui. Le faire-image signifie donc l'implication de l'artiste au sens où celui-ci s'engage dans le réel dans la mesure même où il s'adonne au réel. L'artiste ne s'oriente donc pas vers le réel à travers l'image (comme ce pourrait être le cas s'il s'agissait d'un rapport au monde intentionnel), mais il est immergé au sein même du monde dans l'image. L'implication de l'artiste dans l'image est une activité mêlée de passivité, un "laisser » engageant. Si le premier écueil sous-jacent à la peinture (et donc au performatif de l'image) est l'illusion de l'artiste qui croit pouvoir se tenir hors du monde afin de le peindre de l'extérieur, qui croit donc pouvoir échapper à l'emprise de l'image et en devenir le seul maître, un second écueil est lié à cette immersion première dans le monde. Ce moment infra-pictural de l'artiste plongé dans le monde constitue à la fois la chance de la peinture, qui pourra - peut-être - dire la vérité, et sa menace la plus extrême, car l'artiste pourrait aussi ne pas parvenir à exprimer cette image originaire, à la retenir dans son tableau, et la laisser s'éteindre dans l'abîme.

De même que l'artiste doit se laisser modeler par l'image, il faut aussi qu'un observateur potentiel puisse rejoindre les "sensations confuses» que le peintre exprime sur la toile, semblablement au cocher de Cézanne, qui semblait comme contaminé par l'enthousiasme extatique du peintre. Maldiney cite cette anecdote :

Lorsque Cézanne allait au motif, que de fois, m'a raconté son cocher, il se dressait brusquement dans la voiture, prenait le bras de l'homme. "Regardez... ces bleus, ces bleus sous les pins... Ce nuage là-bas... » Il rayonnait d'extase et l'autre qui n'apercevait que des arbres, du ciel, pour lui toujours les mêmes, ressentait pourtant, m'avouait-il, comme une vague force, une émotion l'envahir et qui lui venait de Cézanne debout, transfiguré, les mains nouées à son épaule, et tout plein d'une évidence qui les sanctifiait ${ }^{23}$.

14 L'image doit donc réaliser cette communion entre l'artiste et le réel, mais aussi entre l'observateur (le spectateur) et l'artiste. Toutefois, nous verrons que cette communion n'est jamais une fusion, et que l'image est toujours en partie manquée par l'observateur, mais aussi par l'artiste lui-même. Nous y reviendrons.

Si une communication est possible par l'image entre l'artiste et l'observateur, c'est parce que le style de l'image est fondamentalement celui du monde lui-même. C'est pourquoi le bleu vibrant et remuant de la Méditerranée peut devenir, sur la toile de Renoir, la couleur d'un ruisseau d'eau douce où se baignent des femmes nues :

[...] on ne demande à la mer que la manière qu'elle a d'interpréter la substance liquide [...]. On peut faire de la peinture en regardant le monde parce que le style qui définira le peintre pour les autres, il lui semble le trouver dans les apparences mêmes (en tant, bien entendu, qu'elles sont apparences siennes) ${ }^{24}$.

16 Tout est dit ici : le style du peintre, qui est en fait le style du monde, n'est pas à copier, imiter ou reproduire, mais à incarner, à vivre depuis l'image originaire de notre rapport premier au monde. 


\section{L'événement performatif du faire-image, ouverture à la pluralité des sens}

17 Le faire en peinture, le faire-image n'est donc pas un acte, qui suppose quelqu'un qui agit et quelque chose qui est agi, mais c'est un événement, qui bouleverse tous ses participants, et un événement performatif, qui se fait dans l'image. Le faire de l'image se réalise comme l'événement qui advient entre l'interrogation que l'artiste adresse au monde, la figure que lui propose le monde, et de nouveau la réponse de l'artiste à ce que le monde lui offre, qui peut figer cette figure du monde en la représentant ou tenter de l'exprimer, réponse de l'artiste prolongée par la réponse du spectateur, qui peut se laisser regarder, se laisser concerner par l'image ou refuser d'y entrer. Monde, artiste et observateur sont ainsi réunis dans l'événement du faire-image. Le terme d'événement désigne ici non pas quelque chose de sensationnel ou de spectaculaire, mais il se réalise en un double sens, que nous reprenons d'une part de Merleau-Ponty, d'autre part de Maldiney. Pour Merleau-Ponty, le peintre emprunte son style au monde en prêtant au monde son corps. Le style est en cela l'expression de l'entrelacs entre le corps et le monde, l'expression même de l'image originaire. Il y a donc événement de l'image par l'instauration de l'incarnation comme rapport au monde qui précède toute dualité entre un sujet et un objet, rapport au monde où je suis d'emblée ouvert à l'autre et au monde, éclaté hors de moi. Pour Maldiney, l'événement est plus proche de sa figure derridienne. Il désigne l'irruption soudaine et imprévisible de «l'événement d'une sensation ", qui " déchire la trame de l'attente et des possibles » ${ }^{25}$. Pour Maldiney, les sensations manifestent la " soudaine morsure de la Réalité $»^{26}$ que subit le peintre. Dans les deux cas, cependant, l'événement indique bien cette dimension pathique qui soustend l'image, et qui suppose aussi un manque et une souffrance inhérents à l'image originaire, et constitutifs de la performativité, nous y reviendrons dans la dernière partie.

18 Le faire-image est donc événement au sens où il fait advenir un monde neuf en rompant avec les apparences ininterrogées : l'image crée du réel en fondant à nouveaux frais sa vérité. En cela consiste sa performativité. Cet avènement d'un monde, par l'incarnation ou la sensation, est aussi l'ouverture d'un sens nouveau, et le sensible est toujours en même temps herméneutique. C'est pourquoi, comme l'écrit Maldiney, "[l]'art est la vérité du sentir, non le mémorial des impressions » ${ }^{27}$. Le terme de " vérité » suggère aussi ce sens qui est créé par l'image. En cela, l'événement du faireimage est bien un dire : il dit du sens, il profère un autre possible, qui n'est cependant pas irrationnel ou magique, mais qui instaure une autre forme de signifiance ne reproduisant pas celle d'un référent hors image. La vérité de l'image n'est donc pas l'adéquation de l'image à un réel qui se situerait hors d'elle, elle n'est pas non plus un idéal situé dans un monde épuré, de part en part imaginaire, en quelque sorte purifié du réel. La vérité de l'image est le sens qui émerge de l'image, qui caractérise son autonomie, et qui a trait à son rythme et à son style propres. La vérité de l'image désigne donc la cohérence qui relie les différentes composantes de l'événement, la cohérence qui est celle de l'image elle-même, mais aussi celle de l'existence de l'artiste et de l'observateur qui se ressaisissent dans le faire-image.

19 Mais ce sens n'est pas inventé ex nihilo par le peintre, car il ne fait qu'extraire du monde des significations qui s'y trouvaient déjà, encore inexprimées. En d'autres termes, disant la vérité, le peintre donne la parole au monde : quand l'image se fait, le monde se 
dit. En effet, comme le montre Merleau-Ponty, chaque fragment du monde recèle en lui "un nombre illimité de figures de l'être ${ }^{28}$, mais le plus souvent, les différents événements de sens, comme le soutient Heidegger, de même que les différents événements des sens, comme l'explique Maldiney, entrent en conflit les uns avec les autres, dans une concurrence violente par laquelle ils s'annulent alternativement. Mais Merleau-Ponty a montré que l'incarnation, une incarnation en elle-même signifiante, est précisément l'intégration de ces incompossibilités, puisque chaque corps empiète sur le tout, est entremêlé au tout sans pourtant fusionner intégralement avec lui. L'incarnation est l'événement de ce processus d'incompossibilisation et d'intégration à la fois. Et la vérité de l'image est la vérité de cette incarnation et en même temps, la vérité du sens. Ainsi, la vérité que dit l'image, à la fois sensible et herméneutique, est une vérité de profondeur, une vérité qui nous fait décoller de la surface de l'apparaître pour nous faire entrer dans la profondeur d'autres possibles. Cette vérité est par définition non univoque, puisqu'elle est l'ouverture à la pluralité des sens. L'image est donc l'événement performatif de l'ouverture de possibles illimités, qui peuvent coexister et cohabiter sans devoir s'annihiler.

Par conséquent, ces possibles sont aussi synesthésiques, et l'image n'est performative que si elle fait intervenir non seulement la vue, mais aussi les autres sensorialités. Car la « vision initiale » n'est pas, on l'a dit, l'acte perceptif de la vue et le peintre doit bien plutôt oublier ce qu'il a saisi visuellement, pour laisser s'exprimer ce qui le touche et le regarde, au sens propre comme au sens figuré. Dans l'image, nous ne voyons plus la propriété ou la qualité d'un objet identifiable - le jaune des tournesols, le vert de l'herbe -, mais une pluralité de sensations nous advient. Voilà aussi ce que l'image fait, ce qu'elle nous fait.

\section{Les risques et échecs possibles de la performativité de l'image}

21 L'image n'est pas création achevée de vérité, mais elle est promesse de création de vérité. Par conséquent, elle peut échouer. Pour que l'événement de l'image puisse avoir lieu, il faut que nous - artiste ou observateur - parvenions à épouser le rythme du réel, c'est-à-dire le rythme de l'image originaire. Mais ce rythme de l'image, ou ce style de l'image, nous l'avons toujours déjà perdu. Et lorsque nous tentons de le retrouver, nous ne le retrouvons que partiellement, si bien qu'un creux, un manque perdure dans l'image. L'image picturale, de même, n'exprime toujours que des traces de cette vérité originaire. Ces traces ne sont pas une déficience de l'image, mais plutôt l'expression que cette vérité n'est elle-même pas transparente, mais opaque et floue. Notre " insertion première [...] dans le vrai ", c'est-à-dire notre enlacement à la chair du monde, est elle-même empreinte de manque, parce que nous n'avons pas prise sur elle, elle nous échappe en partie. La chair n'est pas une étoffe pleine, mais un tissu troué d'absence et de vide. En effet, notre expérience première du monde et d'autrui est d'emblée discordance. La réversibilité est toujours imminente, mais toujours aussi manquée. « [L]'originaire éclate ${ }^{29}$, lit-on dans Le Visible et l'invisible.

Pourtant, comme l'écrit Maldiney : « Le propre d'un rythme est de s'accomplir à travers ses propres failles $»^{30}$. La performativité ne peut donc se réaliser adéquatement que si elle accepte en elle ces fissures, que si elle s'accomplit dans ces interstices. Ces béances de l'image constituent sa menace et son risque autant que sa promesse de réussite. Car 
ce que dit l'image en se faisant image par delà ses propres failles est précisément l'ambiguïté de notre être-au-monde le plus originaire, son étrangeté fondamentale. N'est-il pas en effet profondément dérangeant et déstabilisant que jamais, nous ne sachions ce que nous sommes exactement, puisque tout ce que je touche, vois, goûte, entends et sens, fait partie de moi et n'en fait pas partie, est moi et n'est pas moi ? N'est-il pas tout à fait inquiétant que par mon corps même, par ce qui m'est le plus intime, je sois entièrement livré au monde, sans jamais pouvoir me réfugier en un moi clairement délimité de l'extérieur, puisque d'emblée, par son incarnation même, ce moi est devenu le monde et est devenu autrui ?

L'étrangeté est donc constitutive de l'image, comme le montre Blanchot en rapprochant l'image de l'imago romaine, l'effigie moulée en cire des morts ${ }^{31}$ : l'image est comme la dépouille mortelle, ressemblance dissemblante du vivant, elle est cette absence elle-même, elle est la présence d'une absence. Et de la sorte, "l'homme est défait selon son image $\aleph^{32}$, image qui fonde cette identité sans coïncidence avec soimême. Cette étrangeté angoissante tient aussi à ceci que l'image est toujours miroir : je suis dévisagé dans l'image par cela même que j'envisage. Ainsi, l'étrangeté de l'image est double : elle est celle que je suis à moi-même, mais elle provient aussi du regard inquisiteur de l'autre qui habite chacun de mes faits et gestes. Dans l'image, dans mon image ou l'image que je suis, je suis en même temps scruté et dévisagé par le regard d'autrui, le regard des choses et du monde dans son ensemble. Et ce regard de l'autre est un regard qui me somme à l'éthique, qui m'oblige à la responsabilité et qui m'interdit la fuite. C'est un regard qui fait de mon être, de mon être-image, de mon incarnation sensible et signifiante, une habitation nécessairement éthique. Plus elle se fait, plus elle me fait quelque chose, plus aussi elle me défait, plus l'image dit cette vérité étrange et inquiétante que je suis en étant au monde et aux autres.

Le risque le plus grand pour l'image est donc plutôt celui d'effacer cette discordance originaire, de chercher à l'étouffer. La performativité de l'image échoue véritablement lorsque l'image ne peut plus faire cette incarnation qu'en tant que corps, elle est pourtant. Il y a échec lorsque je ne suis plus capable de soutenir l'image, lorsque je refuse d'habiter cette image que je suis, lorsque je me soustrais au miroir, lorsque mon " image interne », comme disait Ortega, ne peut plus avoir lieu ni advenir. Il semble que ce soit précisément ce que Maldiney essaie de décrire lorsqu'il évoque la pathologie mentale. Les fantasmes, les hallucinations, les délires ainsi que les rêves des malades témoignent le plus souvent de leur impossibilité à habiter leur corps. Les malades ne sont plus capables d'être leur image originaire, parce qu'ils ne peuvent plus supporter ce que celle-ci fait, ce qu'elle leur fait. Les malades se mettent à distance de leur corps, comme c'est tout particulièrement le cas dans la schizophrénie. Ne pouvant plus habiter ce cadre originaire, qui sous-tend chacune de nos actions quotidiennes, ils sont contraints de le réinventer à chaque acte, mais ce faisant, ils ne peuvent que le reconstruire sous forme de représentations mentales plus ou moins figées. La performativité de l'image originaire, pour eux, semble irrémédiablement perdue.

Mais la performativité de l'image échoue aussi en un autre sens, lorsque l'image est soumise à des critères discursifs qui lui sont impropres. Et c'est là que se joue notre responsabilité. Car dans cette conception, l'image est performative avant le langage, ou, plutôt, le langage ne peut être performatif qu'à la manière de l'image, dans la mesure où l'image est notre mode le plus originaire d'être-au-monde, d'habiter le monde. La performativité du langage philosophique doit selon nous être celle de l'image, c'est-à- 
dire une performativité ouverte, qui initie et relance continuellement le mouvement de la pensée au lieu de l'arrêter. Une telle performativité est ce qui garantit et entretient la vie même de la philosophie, sa fécondité qui ne se réduit pas à une utilité. Or, nous pensons qu'on peut caractériser l'usage du langage philosophique chez le dernier Heidegger de performatif au sens de la performativité de l'image. Dans la dernière version datant de 1974 d'un texte sur Cézanne, ce n'est plus la « la coappartenance de la poésie et de la pensée [Zusammengehören des Dichtens und des Denkens] $»^{33}$ qui vient clore le texte, comme c'était le cas dans la version de 1970, mais la mention de «l'imager et de la pensée [Bildens und Denkens] $\aleph^{34}$. Voilà un indice de l'importance de l'image pour le dernier Heidegger même si - sauf dans ce texte - l'image est chez lui peu thématisée en tant que telle.

L'image du Sein barré ( 1955 , est un exemple tout à fait révélateur de ce langage-image ${ }^{35}$. Ce qui est performatif ici, ce n'est pas le mot en lui-même, mais c'est bien l'image du mot, avec les lignes qui viennent le barrer mais surtout l'ouvrir aux différentes dimensions du monde. Car la croix, Heidegger le dit explicitement, n'est pas la simple biffure de l'être, elle montre plutôt en direction de possibles dont la pluralité ne peut pas être définie à l'avance, mais seulement annoncée par ces lignes qui se croisent mais aussi s'écartent les unes des autres sans jamais s'arrêter définitivement. Il est important en effet que ces lignes ne soient pas des segments mathématiques, mais des droites, voire des vecteurs donnant une direction. Ainsi, dans la mesure où le terme d'être, chez Heidegger, désigne justement le rapport entre l'être humain et le monde, on peut voir dans ce Sein barré une expression de l'image originaire au sein même du langage. Le Sein barré est une manière de faire avec les mots comme on peut dire avec l'image. En un sens, il est un peu l'effigie de la performativité de l'image qui s'incarne dans le langage.

\section{Conclusion : une performativité toujours à recommencer}

Si « le langage est la maison de l'être $»^{36}$, et non pas sa prison, c'est bien parce que le langage est, fondamentalement, une forme de l'image originaire par laquelle nous habitons le monde. Il doit laisser se déployer en lui notre rapport au monde sans chercher à l'enfermer dans des catégories trop figées. Ainsi, que la performativité de l'image s'incarne dans l'image picturale ou dans le langage philosophique, à aucun moment, la performativité ne signifie l'accomplissement d'un faire transparent, clos et achevé. Car s'il s'agit de « dire la vérité » de notre être-au-monde dans sa modalité la plus originaire, cette vérité ne peut être qu'une recherche, elle est la tentative de retrouver le déchirement qui caractérise notre contact premier avec le monde. Ce déchirement est à la fois la menace de la performativité, car il peut mener au mutisme complet, à la fin de toute parole, mais aussi et d'abord sa chance, son moteur même. Et cette chance est aussi pour nous la promesse que nous pouvons faire, de chercher, dans notre dire, une expression de cette image originaire.

27 Ainsi la performativité de l'image nous permet de repenser l'apparaître originaire en deçà de toute corrélation fondée sur deux pôles séparés, comme événement intégrant en lui différentes dimensions et impliquant l'engagement de l'humain. Elle signale comment le voir et l'apparaître s'unissent en un dire, c'est-à-dire en une production de 
sens qui peut être exprimée dans l'image picturale ou dans le langage philosophique. L'image fait donc advenir du réel en instaurant un certain rapport de sens. Mais, contrairement aux actes de langage performatifs dans la conception austinienne, il y a performativité lorsqu'un certain risque inhérent est pris en charge et assumé, ce qui ne veut pas dire surmonté, mais accepté. Il y a donc bien, comme chez Austin, superposition d'un faire et d'un dire, mais ce faire et ce dire ne visent pas une réussite qui viendrait s'ajouter de l'extérieur. La performativité de l'image fonctionne dès lors qu'elle s'accomplit, dès lors que le faire est un dire et que le risque est assumé. Mais ce fonctionnement n'est pas à classer du côté d'un fonctionnement "heureux » par opposition à un fonctionnement «malheureux » : le performatif n'est jamais clos ni achevé, il est toujours à faire et à recommencer. Il n'est qu'un geste, celui de l'image originaire, qui nous indique une façon d'habiter plus originairement le monde - que ce soit par la pensée, par l'art, ou par une manière de conduire notre existence.

\section{NOTES}

1. J.-L. Austin, How to do Things with Words, Oxford, New York, Oxford University Press, 1962. Trad. par G. Lane, Quand dire, c'est faire, Paris, Seuil, 1970.

2. Voir P. Bourdieu, Ce que parler veut dire : L'économie des échanges linguistiques, Paris, Fayard, 1982.

3. Voir J. Butler, Gender Trouble: Feminism and the Subversion of Identity, New York, Routledge, 1990. Trad. par C. Kraus, Trouble dans le genre : pour un féminisme de la subversion, Paris, La Découverte, 2005. Voir aussi J. Butler, Excitable Speech: a Politics of the Performative, Londres, Routledge, 1997. Trad. par C. Nordmann, Le pouvoir des mots : politique du performatif, Paris, Ed. Amsterdam, 2004.

4. Voir notamment G. Girot, "Le théâtre performatif entre théâtre du réel et réel du théâtre ", Extension du domaine de l'art, juin 2015, Paris, France (hal-01492767) ; A.-M. St-Jean Aubre, « Entre théâtralité et performativité ", Jeu, n¹55, 2015, p. 64-67.

5. Voir par exemple D. Hantelmann, How to do things with art: the meaning of art's performativity, Zurich, JRP, Ringier, Les Presses du Réel, 2010.

6. Voir J.-L. Austin, How to do Things with Words, op. cit., p. 5, trad., p. 41.

7. Ibid., p. 14 sq., trad., p. 49.

8. J. Derrida, « Passe partout », in La vérité en peinture, Paris, Flammarion, 1978, p. 7.

9. Ibid., p. 8.

10. J. Ortega y Gasset, Vitalité, Âme, Esprit, trad. par P. Posada Varela, in Annales de Phénoménologie, nำ10, 2011, p. 195-223.

11. Ibid., p. 200.

12. Ibid., p. 199.

13. M. Merleau-Ponty, "La perception d'autrui et le dialogue ", in La Prose du monde, Paris, Gallimard, 1969, p. 193.

14. Idem.

15. Et il n'est pas davantage le performatif d'un « acte d'image ». En cela, nous nous écartons de la conception de Bredekamp dans son livre intitulé Der Bildakt : Frankfurter Adorno-Vorlesungen 2007, Berlin, Wagenbach, 2015 [2010]. 
16. Cité par H. Maldiney, «Le faux dilemme de la peinture : abstraction ou réalité », in Regard Parole Espace, Paris, Cerf, 2012, p. 49.

17. Idem.

18. H. Maldiney, «L'esthétique des rythmes », in Regard Parole Espace, op. cit., p. 208.

19. H. Maldiney, «Le faux dilemme de la peinture : abstraction ou réalité », op. cit., p. 48.

20. H. Maldiney, « L'esthétique des rythmes », op. cit., p. 207.

21. H. Maldiney, «Le faux dilemme de la peinture : abstraction ou réalité », op. cit., p. 48 [Nous soulignons.].

22. Ibid., p. 49.

23. H. Maldiney, ibid., p. 48 [Nous soulignons.].

24. M. Merleau-Ponty, « Le langage indirect », in La Prose du monde, op. cit., p. 88.

25. H. Maldiney, « L'art et le rien », in Art et existence, op. cit., p. 195.

26. H. Maldiney, « Le faux dilemme de la peinture : abstraction ou réalité », op. cit., p. 47.

27. H. Maldiney, «Cézanne et Sainte-Victoire : peinture et vérité », in L'Art, l'éclair de l'être, Paris, Cerf, 2012, p. 27.

28. M. Merleau-Ponty, « Le langage indirect », op. cit., p. 85.

29. Ibid., p. 163.

30. H. Maldiney, «L'art et le rien », op. cit., p. 206.

31. Voir M. Blanchot, « Les deux versions de l'imaginaire », in L'Espace littéraire, Paris, Gallimard, 1955, p. 265-277.

32. Ibid., p. 273.

33. M. Heidegger, "Gedachtes ", in Gesamtausgabe [GA], vol. 13, Aus der Erfahrung des Denkens (1910-1976), éd. par H. Heidegger, Francfort-sur-le-Main, Klostermann, 1983, p 223. Cette version date de 1970.

34. M. Heidegger, « Cézanne (spätere Fassung 1974) », in GA 81, Gedachtes, éd. par P.-L. Coriando, Fancfort-sur-le-Main, Vittorio Klostermann, 2007, p. 347.

35. Sur l'émergence d'une phénoménologie de l'image chez Heidegger, nous nous permettons de renvoyer à notre propre article : S. Gourdain, «Le retrait, la dissimulation et l'inapparent : vers une phénoménologie de l'image?", in Heidegger aujourd'hui. Actualité et postérité de sa pensée de l'événement, éd. par S.-J. Arrien, C. Sommer, Paris, Hermann, à paraître.

36. M. Heidegger, Der Satz vom Grund, GA 10, éd. par Petra Jaeger, Francfort-sur-le-Main, Vittorio Klostermann, 1997, p. 143, trad. par A. Préau, Le principe de raison, Paris, Gallimard, 1962, p. 210. 